Int. J. Speleol. 10 (1978), pp. 293 - 302

\title{
Prime osservazioni in margine a un'esperienza di concrezionamento di alabastri calcarei in ambiente ipogeo
}

\author{
di
}

\author{
P.FORTI*, R.CASALI**, G.PASINI***
}

First observations on deposition of calcite sinter in a cave

\author{
SUMMARY
}

Optical analyses of thin slides of case formations carried out by a number of researchers showed a fine structure consisting of a uscession of double laver (thickness: less hath a lew lens of $\mu$ m). In each two layer unit, one is thicker, transparent and composed by an assembly of calcite needle shaped crystals oriented perpendicularly to the layer; the other layer is opaque, cryptocristalline or amorphous.

According to some $\mathrm{C}-14$ dating of archaeological remains covered by the formations each couple of layers would correspond to one year.

In order to check this hypothesis an experiment was set up in the Novella Cave (near Bologna) under controlled conditions. Cave pearls were choosen for investigation owing to the possibility of studying one of them without introducing any perturbation on the formation of the calcite sinter on the others. The growth of cave pearls was studied in a glass cell composed of three sections and described in the paper.

In the first one, some holes of different size and depth were made in order to ascertain the influence of these geometrical parameters in function of the distance from the water drips. Small glass spheres (diameter around $1 \mathrm{~mm}$ ) were placed in the holes as deposition nuclei. A cover was placed over this section to collect the water splash only when the water flow was measured, in order to avoid any change in the natural evaporation rate.

The connection with the second section was obtained by a glass tube designed in order to guarantee a suitable mixing of the water. Both temperature and $\mathrm{pH}$ were measured.

The third section consisted of a graduated container used to measure the flow rate which varied from 0.4-0.5- to 500-700 drops/min (a range of about three orders of magnitude).

Air temperature was also measured during the experiment. The chemical composition of both natural and artificial cave pearls was determined. All the data are reported. No difference (with the exception of $\mathrm{Fe}$ concentration) was detected between the natural and the artificial conditions. Therefore after some years of experimentation the growth rate will be determined under controlled conditions.

\section{Introduzione}

Analisi condotte con il microscopio da mineralogia a luce trasmessa su sezioni sottili di alabastri calcarei a struttura laminare di varie grotte, hanno mostrato

\footnotetext{
*Unione Speleologica Bolognese, Cassero di Porta Lame, Bologna, Italia

**Istituto Chimico "G. Ciamician», Via Selmi, 2, Bologna, Italia

***Laboratorio di Geologia Marina, C.N.R., Bologna, Italia
} 
che in molti casi ogni lamina di questi alabastri, di spessore generalmente non superiore ad alcuni centesimi di millimetro, è costituita da due sottolamine o bande, di cui una, di solito più potente e trasparente è formata da cristalli fibrosi di calcite disposti normalmente alla superficie della banda stessa, mentre l'altra, di solito meno potente e più opaca, è criptocristallina o amorfa.

(iià vari autori hanno avanzato l'ipotesi che ogni lamina (o coppia di bande) si formi nell'arco dell'anno (Broecker et al., 1960), e che il fenomeno sia collega10 alle oscillazioni del chimismo del suolo (Duchaufour, 1965). Questa ipotesi sembrerebbe avvalorata dal rinvenimento sotto concrezioni in fase di deposizione attiva di reperti archeologici, la cui datazione al radiocarbonio confermava abbastanza bene l'età attribuita loro contando il numero delle lamine delle concrezioni sovrastanti (Broecker et al., 1960).

É evidente quindi l'importanza che avrebbe la conferma diretta sperimentale dell'annualità delle lamine, per le sue implicazioni di carattere geocronologico, archeologico e paleoclimatico (potendosi risalire facilmente dallo spessore delle sottobande al prevalere di questo o quel periodo nell'anno).

Naturalmente per conoscere il meccanismo chimico-fisico con cui si formano le bande all'interno delle lamine di accrescimento nelle concrezioni di grotta bisogna tener sotto controllo continuo, per un periodo di tempo abbastanza lungo (oscillante tra i 3 e i 5 anni almeno) molti fattori quali la composizione chimica, la temperatura, il pH e la portata delle acque concrezionanti, nonchè la temperatura e l'umidità relativa dell'ambiente in cui avviene il fenomeno, il tutto ovviamente in condizioni di sicuro concrezionamento.

Allo scopo di studiare l'accrescimento degli alabastri calcarei abbiamo iniziato qualche anno fa i lavori per l'installazione di un Laboratorio Sperimentale Sotterraneo nella Grotta Novella (N. 287 E), cavità ricca di concrezioni calcaree che si sviluppa nei gessi messiniani del Pedeappennino bolognese (Casali et al.1971, 1972; Forti, 1973). Il laboratorio, pressochè ultimato, si trova alla base di un'ampio pozzo ad una profondità di una cinquantina di metri dall'ingresso.

In questo laboratorio si studierà in particolare l'accrescimento di pisoliti a nucleo artificiale: la scelta delle pisoliti è dovuta al fatto che si dovranno prelevare periodicamente campioni delle concrezioni in studio e analizzarli in sezione sottile per controllare il livello di deposizione del carbonato e l'eventuale formazione di bande distinte.

Le pisoliti, uniche fra tutte le concrezioni, consentono questo tipo di prelievo periodico, senza che con questo si alteri minimamente il concrezionamento di quelle che restano, permettendo quindi in definitiva di considerare l'analisi successiva su elementi differenti come compiuta sempre sul medesimo elemento.

Per poter seguire poi in continuo, senza altresı in alcun modo alterarli, i principali fattori che possono influenzare il concrezionamento delle pisoliti è stato 
appositamente progettato, realizzato e sperimentato uno strumento particolare.

Nel presente lavoro vengono riportate la descrizione di tale strumento attualmente in funzione nel laboratorio (Fig. 1), nonchè i primi risultati parziali relativi allo studio delle costanti chimico-fisiche dell'ambiente in cui il concrezionamento avviene e l'analisi chimica delle prime croste di concrezione formatesi attorno ai nuclei artificiali.

\section{Descrizione della vaschetta di concrezionamento}

Per provocare la formazione di pisoliti si sono collocate delle palline di vetro del diametro di mm 2,5 circa (che avrebbero dovuto funzionare da nuclei) all'interno di una vaschetta di vetro appositamente studiata, sistemata in corrispondenza di uno stilicidio perenne di acque sicuramente concrezionanti.

Come materiale da costruzione è statc scelto il vetro a causa della sua trasparenza, resistenza chimica e duttilità.

La vaschetta (fig.2) è stata costruita in tre sezioni separate, collegate tra loro da giunti sferici: il motivo per cui si è preferito costruirla a sezioni piuttosto che tutta di un pezzo è duplice. In primo luogo, in caso di rottura, non improbabile a causa delle possibili cadute di piccoli sassi nel pozzo alla cui base la vaschetta è posta, risulterebbe più semplice la sostituzione di una sola sezione; in secondo luogo in questa maniera le varie operazioni di misura risultano facilitate.

Passando quindi a considerare le tre sezioni in dettaglio si deve notare che la prima (A) costituisce la vaschetta vera e propria in cui avviene il concrezionamento delle pisoliti. Il fondo di questa sezione è modellato in modo da formare un certo numero di sottovaschette, di profondità e distanza dal centro variabile, per poter studiare l'influenza della profondità e della distanza radiale dal punto di impatto della goccia cadente (circa al centro della vaschetta) sulla deposizione di carbonato di calcio sulla superficie delle pisoliti.

La sezione $A$ è dotata di un «coperchio» mobile (4 in fig. 2 ) che può servire eventualmente a intercettare gli schizzi provocati dall'impatto delle gocce e a convogliarne l'acqua in una grondaia (2) e quindi, attraverso un tubo flessibile (3-8), nella sezione $C$.

Il tubo da cui avviene la fuoriuscita di acqua per tracimamento da questa prima sezione è stato realizzato a $2 \mathrm{~cm}$ dal fondo della vaschetta di vetro, altezza questa che corrisponde a quella che veniva raggiunta dall'acqua nella vaschetta naturale con pisoliti che esisteva precedentemente nel medesimo luogo del laboratorio sotterraneo.

Nella seconda sezione (B) sono posti gli strumenti per la misura del pH e della 


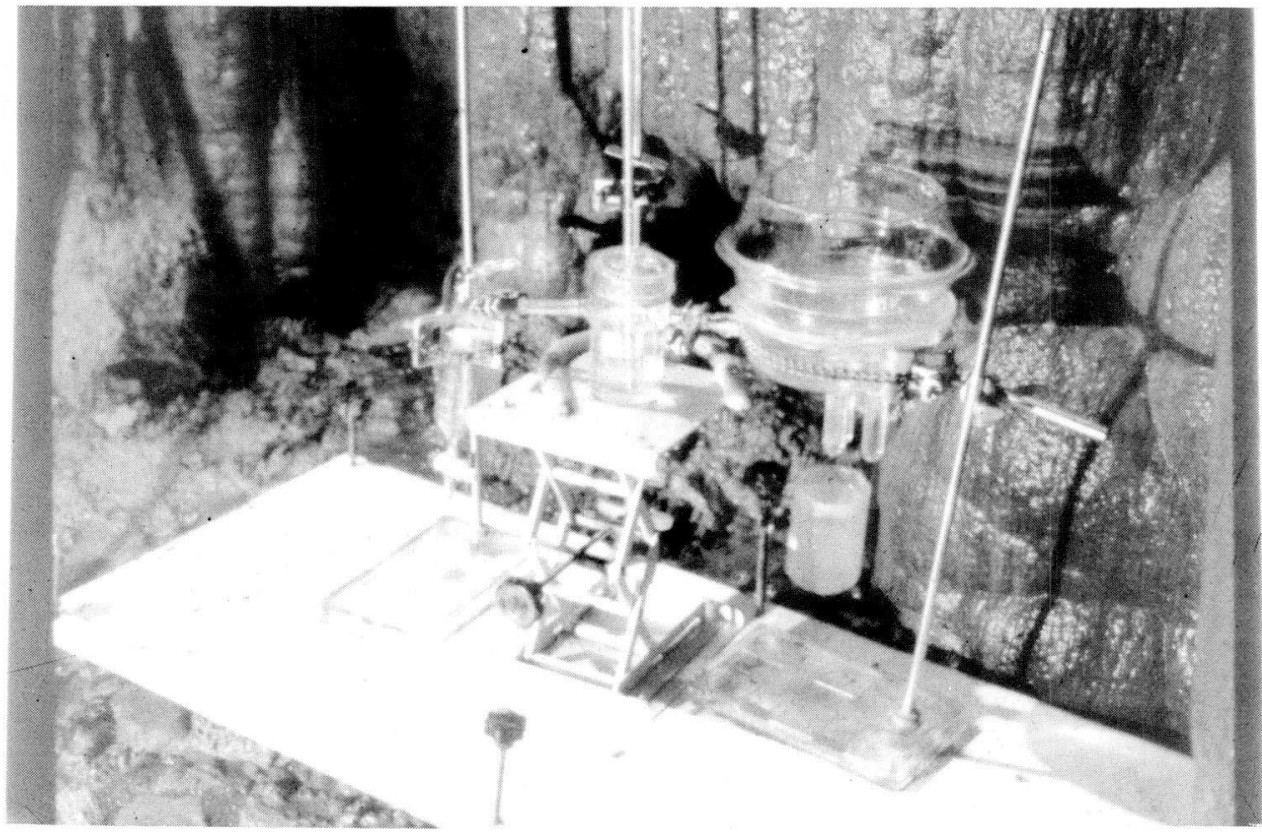

1.ig. I - I a cella in vetro per lo studio dell'accrescimento delle pisoliti in sede alla base di un pozzo di 2 () metri all'interno della (irotta Novella.

Novella cave: the glass cell for the study of the growth of cave pearls at the bottom of a pit 20 meters deep.

Iï. 2 - Cella di vetro per lo studio del concrezionamento delle pisoliti:

A serione dove avviene il concrezionamento

B serione dove vengono fatte le misure di temperatura e $\mathrm{pH}$

C'serione dove si effettuano misure di portata

1 sottovaschette a profondità variabile

2 doccia convoglia-spruzzi

3 tubo flessibile che connette la doccia alla sezione C

4 coperchio mobile raccogli-spruzzi

5 giunti sferici di connessione

6 microelettrodo bipolare

7 termometro tarato a $1 / 10$ di ${ }^{\circ} \mathrm{C}$

8 Connessioni alla sezione $\mathrm{C}$

Glass cell for the study of the growth of cave pearls.

A Deposition section

B Temperature and $\mathrm{pH}$ measurement section

C Flow rate measurement section

1 Small holes of different depth and radial distance from water drip.

2 Splash collector

3 Connection to 8

4 Movable cover

5 Ball joints

6 Glass-calomel bipolar microelectrode

7 Thermometer

8 Connection from? 


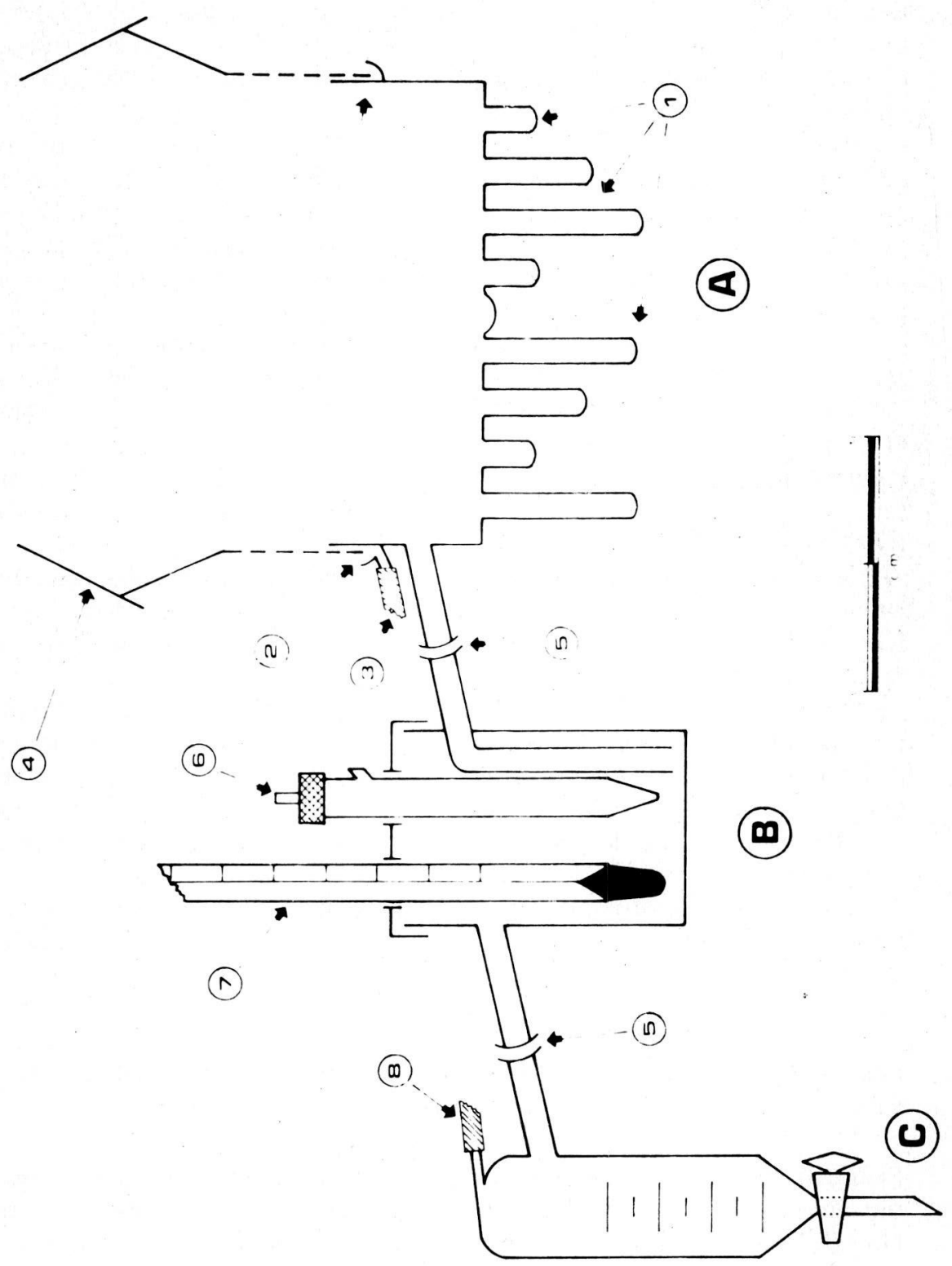


temperatura. Per assicurare il continuo mescolamento dell'acqua, indispensabile al fine di ottenere valori attendibili per le grandezze che si misurano, in questa sezione l'acqua viene fatta entrare presso il fondo ed uscire per tracimamento.

Gli strumenti di misura consistono attualmente in un termometro con scala suddivisa in decimi di gradi centigradi ed in un microelettrodo bipolare collegato ad un pHmetro a pile. L'uscita dalla seconda sezione dell'acqua avviene per tracimamento attraverso un tubo di raccordo che si apre a circa 10 centimetri dal fondo, per far si che il bulbo del termometro e l'elettrodo siano sempre ben immersi.

La terza sezione $(C)$ è stata studiata per avere informazioni nella maniera più semplice e dettagliata possibile sulla portata dell'acqua che cade all'interno della vaschetta, portata che nel corso dell'anno può variare di un fattore $10^{3}$. La sezione consta praticamente di un imbuto separatore, tarato in volumi, il cui rubinetto viene chiuso solo al momento della misura, in modo che, sfruttando la varie tacche di taratura, in tempi relativamente brevi si possono misurare portate da 0,4-0,5 gocce/minuto a 500-700 gocce/minuto.

A questa sezione quindi può arrivare tutta l'acqua che cade nella vaschetta e ne esce per tracimamento come pure quella che ne sprizza fuori dopo l'impatto; quest'ultima, come abbiamo detto, viene raccolta dal "coperchio» nella apposita grondaia.

Le sperimentazioni in grotta, in corso su questo apparecchio da poco tempo, ne hanno confermato le doti di maneggevolezza e funzionalità, permettendo l'inizio di un certo numero di osservazioni sistematiche; l'unico inconveniente per ora riscontrato è quello della dimensione delle sottovaschette nella prima sezione, che risultano un poco anguste quando si vogliono prelevare pisoliti campione da analizzare.

Anche per questo motivo, qualora si rendesse necessaria la costruzione di un altro esemplare di questa apparecchiatura, si dovrebbero fare sottovaschette con sezioni interne maggiori e a fondo piatto.

\section{Primi dati fisici e chimici raccolti nel laboratorio sotterraneo della Grotta No- vella}

Prima di costruire e installare lo strumento sopra descritto sono state condotte per circa un anno in corrispondenza del Laboratorio Sotterraneo della Grotta Novella osservazioni sistematiche su alcuni dei principali fattori che si ritenevano correlati con il fenomeno della deposizione del carbonato di calcio. 
Per compiere tali osservazioni si è collocata in corrispondenza del principale stillicidio una vaschetta di vetro cilindrica a fondo piatto contenente qualche decina di sferule di vetro del diametro di $2,5 \mathrm{~mm}$ circa; si sono inoltre utilizzati due termometri divisi in decimi di grado centigrado e cartine indicatrici ad alta sensibilità (intervallo di $\mathrm{pH}=0,3$ ).

Queste osservazioni dovevano servire anzitutto a confermare che il luogo scel10 per le esperienze di concrezionamento fosse adatto allo scopo (lo stillicidio, ad esempio, doveva essere continuo anche nei periodi meno piovosi) e inoltre dovevano fornire indicazioni utili per il dimensionamento dello strumento. Pur trattandosi di osservazioni molto elementari, abbiamo ritenuto valesse la pena di riportarne $\mathrm{i}$ risultati in questo lavoro (v. Tab. 1).

\begin{tabular}{|c|c|c|c|}
\hline $\begin{array}{l}\text { Data } \\
\text { Date }\end{array}$ & $\begin{array}{c}\text { Temperatura } \\
\text { aria } \\
\text { Air temperature } \\
\underset{{ }^{\circ} \mathrm{C}}{ }\end{array}$ & $\begin{array}{c}\text { Temperatura } \\
\text { acqua vaschetta } \\
\text { Water } \\
\text { temperature } \\
\text { in the cell }{ }^{\circ} \mathrm{C}\end{array}$ & $\begin{array}{c}\text { Flusso } \\
\text { dell'acqua } \\
\text { nella vaschetta } \\
\text { gocce/minuto } \\
\text { Water flow rate } \\
\text { in the cell } \\
\text { (drops } / \text { min) }\end{array}$ \\
\hline $6-X 1-1971$ & & 9. & 2 \\
\hline 13 & & 9. & 11 \\
\hline 21 & & 8.9 & 31 \\
\hline 28 & & 9.3 & 270 \\
\hline 5 -XII- & & 8.9 & 70 \\
\hline 12 & & 8.7 & 38 \\
\hline 19 & & 8.7 & 25 \\
\hline $9-1-1972$ & & 8.6 & 71 \\
\hline 23 & & 8.5 & 131 \\
\hline 30 & & 8.6 & $>300$ \\
\hline $5-11$ & & 8.7 & $\gg 300$ \\
\hline $5-111$ & & 8.8 & 275 \\
\hline 26 & & 8.8 & 190 \\
\hline $9-I V$ & 8.7 & 8.7 & 90 \\
\hline 16 & & 8.6 & 65 \\
\hline 23 & 9. & 9.1 & $>300$ \\
\hline $1-V$ & 9. & 9.2 & $>300$ \\
\hline 14 & 9.2 & 9.2 & 270 \\
\hline 25 & 8.9 & 9.1 & 155 \\
\hline $11-\mathrm{VI}$ & 8.8 & 9.0 & 34 \\
\hline 25 & 8.8 & 9.0 & 24 \\
\hline 9-VII & 8.9 & 9.0 & 17 \\
\hline 23 & 8.9 & 9.1 & 12 \\
\hline 15-VIII & 9. & 9.1 & 11 \\
\hline 3-IX & 9. & 9.1 & 11 \\
\hline
\end{tabular}

Dati relativi all'ambiente in cui vengono compiute le esperienze di concrezionamento. Data of the environment of the deposition experiment 
Esaminando la Tab. 1 si può notare anzitutto che la temperatura dell'aria nell'ambiente in cui avviene il concrezionamento è sempre molto prossima a quella dell'acqua che cade nella vaschetta, differendone al massimo di $0,2^{\circ} \mathrm{C}$; si può notare inoltre che la temperatura dell'aria è quasi sempre leggermente . inferiore a quella dell'acqua; dai dati disponibili si può desumere anche che l'escursione termica dell'aria e dell'acqua nel Laboratorio Sotterraneo non supera quasi certamente il grado centigrado.

Per avere un'idea della portata delle acque concrezionanti e delle sue variazioni nel tempo si è contato di volta in volta il numero delle gocce che cadevano nella vaschetta nell'arco di un minuto, non essendo stato ancora progettato il sistema di misurazione appena descritto per la nuova vaschetta.

I valori riportati in tabella, anche se non coprono interamente l'arco di un anno, indicano con sufficiente chiarezza l'esistenza di due distinti periodi di analoga durata: periodo di piena invernale che va dalla fine di Novembre alla fine di Maggio, ed uno di magra estiva che va da Giugno a Novembre.

Questi dati di portata, pur riferendosi praticamente a un solo anno, danno certamente un'idea abbastanza esatta del regime annuo dello stillicidio nel Laboratorio Sotterraneo della Grotta Novella, che è analogo, come ci si poteva aspettare, a quello dei fiumi della zona in cui si apre la grotta.

È possibile che i due periodi, di magra e di piena, siano da mettere in relazione, assieme ad altri fattori, col diverso tipo di deposizione del carbonato di calcio nelle due bande di ciascuna lamina di concrezione.

Naturalmente solo esperienze più sofisticate potranno confermare, questa ipotesi. Durante lo stesso periodo in cui sono state fatte le osservazioni di temperatura e di portata è stato anche controllato il grado di acidità $(\mathrm{pH})$ delle acque concrezionanti.

Misure periodiche del pH sono state effettuate usando, come si è detto, cartine indicatrici ad alta sensibilità. Confrontando di tanto in tanto i dati cosı ottenuti con quelli forniti da un pHmetro portatile a batteria si sono però rilevate sensibili differenze; queste sono dovute probabilmente al fatto che le cartine indicatrici possono presentare un grado di precisione alquanto basso nei confronti di soluzioni tamponate, come possono essere considerate le acque ipogee interessate dall'equilibrio dell'acido carbonico.

Pertanto non possiamo ancora fornire purtroppo dati attendibili sull'andamento del pH delle acque concrezionanti nel corso dell'anno, fattore che quasi certamente ha un'importanza rilevante nella formazione delle due diverse bande di ciascuna lamina alabastrina. Possiamo solo dire, in base alle misure effettuate col pHmetro portatile, che il pH delle acque concrezionanti nel Laboratorio Sotterraneo della Grotta Novella presenta variazioni stagionali in un intervallo compreso tra 6,5 e 8,5 . 
Analici chimica percentuale di campioni di croste alabastrine sia naturali che formatesi all'interno dellat barchellat di betro.

( hemical anals is (wcight $0_{0}$ ) of samples of calcite formations both natural and obtained within the experimental cell.

\begin{tabular}{|c|c|c|c|}
\hline \multirow[t]{2}{*}{$\begin{array}{l}\text { (inmponconti } \\
\text { (inmponconts }\end{array}$} & \multirow{2}{*}{$\begin{array}{l}\text { Concresione } \\
\text { naturale } \\
\text { Natural } \\
\text { formation }\end{array}$} & \multicolumn{2}{|c|}{$\begin{array}{l}\text { Concrezioni nella vaschetta } \\
\text { Formations in the cell }\end{array}$} \\
\hline & & $\begin{array}{c}\text { Campione del } \\
5 \text { giugno } 1973 \\
\text { Sample of } \\
\text { June } 5,1973\end{array}$ & $\begin{array}{c}\text { Campione del } \\
2 \text { luglio } 1974 \\
\text { Sample of } \\
\text { July } 2,1974\end{array}$ \\
\hline ('a( () (calcite) & 81.0 & 81.0 & 82.0 \\
\hline $\mathrm{Fe}$ & 0.29 & 0.02 & 0.09 \\
\hline $\mathrm{SO}_{4}$ & - & - & - \\
\hline $\mathrm{Mg}$ & - & - & - \\
\hline $\mathrm{Al}$ & tracce-trace & tracce-trace & tracce-trace \\
\hline $\begin{array}{l}\text { Mat argilloso } \\
\text { Clay material }\end{array}$ & tracce-trace & tracce-trace & tracce-trace \\
\hline $\begin{array}{l}\text { Insolubile } \\
\text { Not soluble }\end{array}$ & 16.7 & 18.9 & 18.8 \\
\hline
\end{tabular}

\section{Composizione chimica delle concrezioni ottenute.}

Una delle preoccupazioni maggiori all'inizio dell'esperienza era quella di non alterare minimamente l'ambiente concrezionante con l'introduzione, peraltro necessaria, di supporti per la deposizione.

Per questo motivo è stata nostra preoccupazione di analizzare chimicamente le prime croste di concrezione, non appena queste sono apparse sulle sferule del vetro e a distanza di circa un anno, onde appurare se avessero composizione analoga a quella delle limitrofe concrezioni naturali oppure manifestassero differenze significative.

In Tab. 2 sono riportati i risultati delle analisi fatte: la corrispondenza tra le composizioni delle due concrezioni all'interno della vaschetta e di quella naturale è molto buona.

Le analisi chimiche sembrano avere quindi definitivamente fugato il timore della possibilità, peraltro remota, che l'introduzione di una vaschetta e di sferule di vetro nell'ambiente concrezionante ne potesse alterare il processo di deposizione.

\section{RINGRAZIAMENTI}

Si ringrazia l'Istituto Italiano di Speleologia per gli strumenti messi a disposizione.

Si ringraziario inoltre F. Ranuzzi per il contributo dato per le analisi chimiche e L. Minghetti per l'aiuto prestato sia nella progettazione che nella esecuzione della nuova cella in vetro. 


\section{RIASSUNTO}

Vengono riportati i risultati parziali di alcune osservazioni fisiche e chimiche riguardanti le acque di stillicidio, l'aria e le concrezioni di un laboratorio sperimentale sotterraneo per lo studio del significato cronologico delle lamine delle concrezioni alabastrine. Il laboratorio i situato in una grotta nei gessi presso Bologna (Grotta Novella), a circa $50 \mathrm{~m}$ di profondita dall’ingrewo.

Viene inoltre descritta un'apparecchiatura progettata c realizata appositamente per seguire in continuo il fenomeno di accrescimento delle pisoliti.

\section{BIBLIOGIRAFIA}

R. CASALI, C. CENCINI, P. FORTI e R. ZAVATTI - 1971, Sulla necessita di una varionc -perimentale ipogea nell'ambito della valorizzazione Farneto-Croara. Rassegna Speleologica ltaliana., Mem. X, pp. 263-267.

R. CASALI, P. FORTI, G. PASINI e R. ZAVATTI - 1972, II laboratorio yprimentale ipogen «Grotta Novella». Speleologia Emiliana., II, a. IV, (7), pp. 49-54.

P. DUCHAUfOUR - 1965, Prècis de Pédologie, Masson \& C.. Pari

P. FORTI - 1973. Il laboratorio sotterraneo grotta Norclla. Sial. a. I. (1), pl. 32-37.

W.S. BROECKER, E.A. OISON and P.(. ORR - 1960. Radiocarbon Neasurments and Innual Rings in Cave Formations. Nature, vol. 185. 11. $4706.97 .93-94$. 